\title{
A general relation for group delay and the relevance of group delay for refractometric sensing
}

\author{
Hugo J.W.M. Hoekstra, ${ }^{1, *}$ and Manfred Hammer ${ }^{1,2}$ \\ ${ }^{1}$ University of Twente, MESA ${ }^{+}$Institute of Nanotechnology, P.O. Box 217, 7500 AE Enschede, The Netherlands \\ ${ }^{2}$ University of Paderborn, Theoretical Electrical Engineering, Warburger Strasse 100, 33098 Paderborn,Germany \\ *Corresponding author: H.J.W.M.Hoekstra@utwente.nl
}

Received Month X, XXXX; revised Month X, XXXX; accepted Month X, XXXX; posted Month X, XXXX (Doc. ID XXXXX); published Month X, XXXX

The relevance of our definition for sensitivity in refractometric sensing, being the relative change of the transmittance of a certain output channel of an optical device over the change of the refractive index of the probed material, is discussed. It is compared to one based on spectral shift per refractive index unit change. Further, it is discussed how group delay and sensitivity are interrelated, can be converted into each other and which physical quantities are relevant for high sensitivity. As a byproduct of the presented theory a general expression relating group delay and the ratio of the time-averaged optical energy and the input power is presented.

OCIS codes: (130.6010) Sensors; (260.0260) Physical optics; (280.4788) Optical sensing and sensors.

http://dx.doi/org/10.1364/AO.99.099999

\section{Introduction}

The big potential of integrated optical refractometric sensing devices is evidenced by the large number of publications in the field, as is seen in the review papers of, for example [1-2]. Many different device implementations have been considered in the literature. This is related to technological issues and the nature of the process to be monitored on the one hand, but also to the pursuit of devices with a high sensitivity, and so a low limit of detection (LOD), on the other hand. As a measure of sensitivity, scientists often characterize their device by the spectral shift per refractive index unit (RIU) change. An aim of this paper is to elucidate why this is only partly meaningful, as also noted by [3]. To that end a definition for the sensitivity is used [4] that is directly related to the LOD for index changes [4].

The main aim of this paper is to discuss which physical quantities are of relevance for a high sensitivity. A newly derived expression relating the group delays of the output fields of an arbitrary structure to the ratio of the volume integrated time-averaged energy density and input power is presented. It is shown that sensitivity and partial group delay, i.e., the part of the group delay originating from the material to be sensed, can be converted into each other using a simple Mach-Zehnder like interferometric set-up. From the above it follows that a large partial group delay for a certain structure is sufficient to construct a device with large sensitivity. 
The rest of this paper is organized as follows. In section 2 our definition for the sensitivity is presented and its relation with the LOD (for index changes) is discussed, in relation to the definition based on spectral shift per RIU. In section 3 the derivation of the aforementioned general relation for group delay is presented. The relation is illustrated via a simple example in section 4. In section 5 we discuss the impact of this relation in regards to sensitivity and the relevance of group delay for sensitivity. The paper ends with conclusions in section 6 .

\section{Sensitivity in refractometric sensing}

In this section we will introduce and discuss our definition of the sensitivity $S$, assuming that the index changes of a sample material present in the sensing set-up, $n_{s}$, is monitored. It is further assumed that the wavelength $\lambda$ is scanned on sensing. Assuming that the dominant noise is proportional to the transmittance, as a consequence of, for example, power fluctuations of the source, variations in detector responsivity, and mechanical instabilities, the sensitivity is defined by [4]

$$
S=\left|\partial \ln T / \partial n_{s}\right|
$$

where $T$ is the transmittance corresponding to the chosen output and input. As a constraint to the above, $T$ should not be too low as then the above assumption no longer holds, owing to the presence of dark current noise and shot noise. The $L O D$ can be expressed by [4]

$$
L O D=\alpha \sigma_{\ln T} /\left(S_{\max } \sqrt{M}\right)
$$

with $\sigma_{\ln T}$ the standard deviation of the noise, $M$ the number of sampling points and $\alpha$ a proportionality constant (typically $\alpha \sim 2-4$ ) depending on the shape of the response curve. The subscript max indicates that the maximum value over a considered spectral response curve is taken. It is noted that if the signal is diminished by a factor of 2 (with conservation of signal to noise ratio, and therefore of $\sigma_{\ln T}$ ), this leads to an unaltered $S$, and therefore to an unchanged $L O D$, according to the definition (1). A further justification of Eq. (1) follows from considering two identical cascaded sensing devices, which leads to a doubling of the $L O D$, as expected. Definition (1) is our preferred definition of the sensitivity and will be used further on in this paper.

\subsection{An alternative definition of the sensitivity}

Next we will discuss the shortcoming of an alternative definition of sensitivity based upon the spectral shift per RIU often used in the literature. Huge spectral shifts in sensing devices can be achieved via a well-chosen device lay out and parameters. Such devices have the big advantage of relaxing the constraints on spectral resolution, but are not necessarily an improvement with regards to $S$; this is discussed next, using two examples from the literature. 


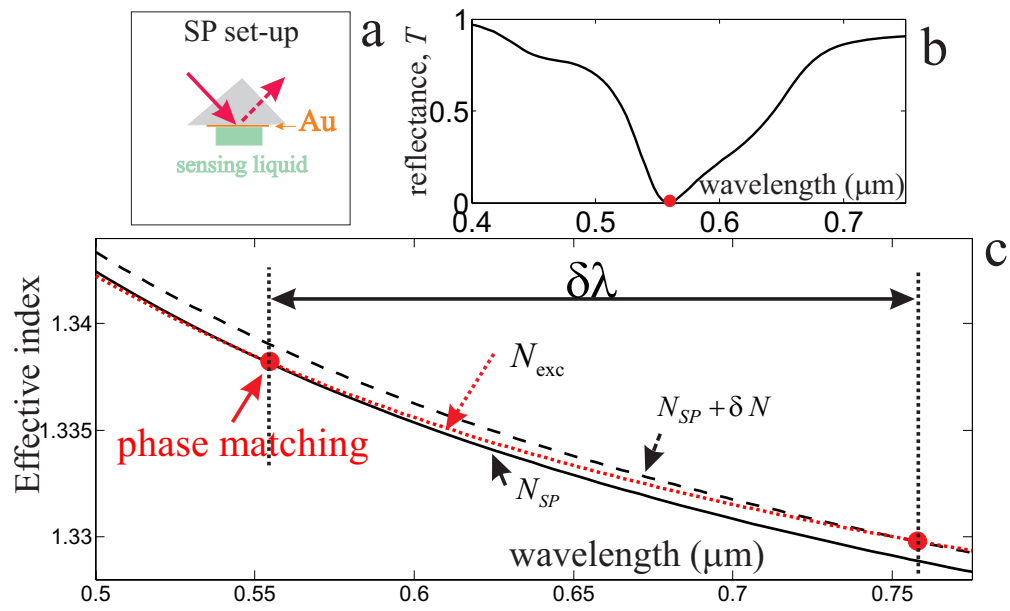

Fig. 1. Illustration of the huge spectral shifts in a SP set-up: a) schematic of the set-up, b) a typical response curve with $R$ the reflectance, and c) idealized dispersion curves for the excitation (dotted line), the SP (solid line), and for the SP after a small modal index change $\delta N$ (dashed line).

\section{A surface plasmon sensing set-up}

It has been shown that surface plasmon (SP) sensing set-ups demonstrate large spectral shifts on sensing [5,6] if the effective index of the SP, $N_{S P}$, is almost equal to the effective index $N_{\text {exc }}$ of the excitation beam, ( $N_{\text {exc }}=n_{p} \sin \theta$, where $n_{p}$ is the dispersive index of the used prism and $\theta$ the angle of incidence at the base of the prism: i.e., prism-sample interface) over a wide wavelength range (see Fig. 1). The resonance wavelength, corresponding to minimum reflectance, is determined by the intersection point of the two curves. Therefore, a small change $\delta N$ of $N_{S P}$, owing to an index change of the sensed medium, leads to a huge shift of the resonance wavelength. However, a second consequence of $\Delta N \equiv N_{S P}-N_{\text {exc }}$ being small over a wide wavelength range is that the reflectance dip is spectrally wide. The two effects counteract each other as expressed by

$$
S=\left|\frac{\partial \ln T}{\partial n_{s}}\right|_{\lambda}=\left|\left(\frac{\partial \ln T}{\partial N_{S P}}\right)_{\lambda}\left(\frac{\partial N_{S P}}{\partial n_{s}}\right)_{\lambda}\right|,
$$

where $T$ is the reflectance, leading to

$$
S=\left|\left(\frac{\partial \ln T}{\partial \lambda}\right)_{N_{S P}}\left(\frac{\partial \lambda}{\partial N_{S P}}\right)_{\ln T}\left(\frac{\partial N_{S P}}{\partial n_{S}}\right)_{\lambda}\right| .
$$

In the above equation the subscript $N_{S P}$ indicates that the considered quantity is constant apart from dispersive changes. The first term at the right hand side of Eq. (4) is inversely proportional to the width of the SP reflectance dip, which itself is inversely proportional to $|\partial \Delta N / \partial \lambda|$. Therefore, the first term is directly proportional to $|\partial \Delta N / \partial \lambda|$. The second term on the right hand side, which corresponds to the spectral shift of (say) the dip for a small change of $N_{S P}$ (on sensing), is inversely proportional to $|\partial \Delta N / \partial \lambda|$, and indeed the two effects of $N_{S P} \approx N_{e x c}$ have no net result for $S$.

A sensing set-up consisting of two cascaded cavities

A second example is the sensing set-up presented and discussed in detail by $[7,8]$, consisting of two cascaded cylindrical cavities such that the transmission combs have a 
slightly different free spectral range. One of the cavities (transmittance $T_{1}$ ) is sensitive to index changes and the second one (transmittance $T_{2}$ ) serves as a filter. As reported [7,8], the total spectral shift of the total transmittance $T=T_{1} T_{2}$ is huge, in particular if the difference between the two free spectral ranges is small. However, it is easy to see that the sensitivity according to definition (1) is identical to that of the sensing cavity alone as $T_{2}$ does not depend on $n_{s}$.

\section{A general relation for group delay}

In this section we will present expressions for an arbitrary photonic structure, which is nonabsorbing and for which there is no spatial overlap between the (different) outgoing and incoming fields that relate the partial group delays of the different output channels to the ratio of the partial electric energy and the input power. The term 'partial' refers to the sample material of the considered structure. These expressions will be used in Section 5 to show the relevance of the above ratio for the sensitivity. As a byproduct of the presented theory a general relation is derived showing that the sum of the (total) group delays weighted by the transmittances of the corresponding output channels is equal to the ratio of the time-averaged energy and the input power.

We consider an arbitrary, non-magnetic $(\mu=1)$ non-absorbing structure with $Q$ independent output modes with corresponding transmittances $T_{m}, m=1, . ., Q$, where $\sum_{m=1}^{Q} T_{m}=1$, excited by light at angular frequency $\omega$ with an input power $P_{i n}$. A time dependence of the form $\exp (i \omega t)$ is assumed. The structure consists of $P$ different dispersive and isotropic materials having refractive indices $n_{j}, j=1, . ., P$, with $\varepsilon_{j}=n_{j}^{2}$ denoting the relative permeability of material $j$. The boundary of the structure should be defined such that it includes all materials that affect the output field for a given input field. For example, evanescent fields of waveguides (WGs) being part of the structure should be well-confined to it. It is further assumed that fields leaving the structure are not re-entering it via reflections, and also that the transmittance for each output mode $m$ can be written as

$$
T_{m}=\left|t_{m}\right|^{2},
$$

with $t_{m}$ denoting the modal transmission coefficient of output channel $m$. The transmission coefficient $t_{m}$ is a complex variable having a phase corresponding to the dominant (say) electric field components of input and output fields.

As mentioned above, the resulting formula will hold for non-absorbing materials. However, for the derivation we will need the introduction of small absorption in the materials of the structure by assuming $n_{l}^{\prime \prime} \lesssim 0, l=1, . ., P$. Thereafter, to obtain the desired expressions, we will consider the limit $n_{l}^{\prime \prime} \rightarrow 0$. Here and in the rest of the paper we denote real and imaginary parts of complex quantities by a single (') and double prime ("), respectively.

For the derivation below it is further required that we may write for the outgoing power

$$
P_{\text {out }}=P_{\text {in }} \sum_{m=1}^{Q}\left|t_{m}\right|^{2},
$$

also for the case that absorbing materials are present. But, as spatially overlapping modes in an absorbing structure are generally no longer orthogonal, using a power related inner product, the above implies that all outgoing modes should be spatially separated from each other and from the incoming mode, such that cross terms originating from different modes do not contribute to the outgoing power. Consequently, each channel supports not more than one mode. So, it is assumed that Eq. (6) also holds if materials of the structure are absorbing.

The group delay [9] of a certain output mode $m$ is given by 


$$
\tau_{g, m}=-\operatorname{Im}\left(\frac{\partial \arg \left(t_{m}\right)}{\partial \omega}\right)=-\operatorname{Im}\left(\frac{\partial \ln t_{m}}{\partial \omega}\right) .
$$

In order to rewrite the right hand side of Eq. (7) we note that, according to wave equations as derived from Maxwell's equations, the quantities describing the behavior of the optical fields in a structure depend on angular frequency and indices according to $f=f\left(n_{1} \omega, n_{2} \omega, \ldots ..\right)$. Here $f$ is a complex analytic function of the product of $\omega$ and the $P$ (generally) different refractive indices, which may vary owing to both material changes and dispersion effects. As a consequence of the dependence of $f$ on $\omega n_{l}, l=1-P$, we may write

$$
d f=\sum_{l=1}^{P}\left(\frac{\partial f}{\partial\left(n_{l} \omega\right)}\right)_{n_{\forall \neq 1} \omega} d\left(n_{l} \omega\right)
$$

leading to

$$
\left(\frac{\partial f}{\partial \omega}\right)_{n_{1-P}}=\sum_{l=1}^{P}\left(\frac{\partial f}{\partial\left(n_{l} \omega\right)}\right)_{n_{\forall \neq l} \omega}\left(\frac{\partial\left(n_{l} \omega\right)}{\partial \omega}\right)_{n_{l}} .
$$

In the above the subscript $n_{\forall \neq 1} \omega$ refers to all materials other than the one labeled with $l$, i.e., the derivative of $f=f\left(n_{1} \omega, n_{2} \omega, \ldots ..\right)$ with respect to only $n_{l} \omega$ is considered while the other products $n_{m} \omega, m \neq l$ are constant. The subscripts $n_{l}$ and $n_{1-P}$ denote that that the indicated indices are constant for a constant frequency (i.e., the corresponding materials do not change) but dispersive changes are taken into account. So, it follows for example:

$$
\left(\frac{\partial\left(n_{l} \omega\right)}{\partial \omega}\right)_{n_{l}}=n_{l}+\omega\left(\frac{\partial n_{l}}{\partial \omega}\right)
$$

Equation (8) can be rewritten using

$$
\left(\frac{\partial f}{\partial n_{l}}\right)_{\omega}=\left(\frac{\partial f}{\partial\left(n_{l} \omega\right)}\right)_{n_{\forall \neq l} \omega}\left(\frac{\partial\left(n_{l} \omega\right)}{\partial n_{l}}\right)_{\omega}=\omega\left(\frac{\partial f}{\partial\left(n_{l} \omega\right)}\right)_{n_{\forall \neq 1} \omega},
$$

leading to

$$
\left(\frac{\partial f}{\partial \omega}\right)_{n_{1-P}}=\sum_{l=1}^{P} G_{l}\left(\frac{\partial f}{\partial n_{l}}\right)_{\omega} ; \quad G_{l} \equiv \frac{1}{\omega}\left(\frac{\partial\left(n_{l} \omega\right)}{\partial \omega}\right)_{n_{l}} .
$$

Taking $\operatorname{Im}(\ln t)$ for $f$ and also using Eq. (7) we arrive at,

$$
\tau_{g, m}=-\operatorname{Im}\left(\frac{\partial \ln t_{m}}{\partial \omega}\right)=\sum_{l=1}^{P} \tau_{g, m}^{l},
$$

with

$$
\tau_{g, m}^{l} \equiv-G_{l} \operatorname{Im}\left(\frac{\partial \ln t_{m}}{\partial n_{l}}\right)
$$

the partial group delay originating from material $l$ for output mode $m$. 
As mentioned above, we consider the effect of a small (negative) imaginary part of the index $n_{l}$, denoted by $n_{l}^{\prime \prime}$, where $l$ labels the material. Starting from the complex Poynting theorem [10]

$$
\nabla \cdot\left(\mathbf{E} \times \mathbf{H}^{*}\right)=-i \omega \mu_{0}|\mathbf{H}|^{2}+i \omega \varepsilon^{*} \varepsilon_{0}|\mathbf{E}|^{2},
$$

integrating the real parts over the volume of the device, $V$, leads to

$$
\int_{V} \operatorname{Re}\left[\nabla \cdot\left(\mathbf{E} \times \mathbf{H}^{*}\right)\right] d \tau=\operatorname{Re}\left[\int_{\text {surface }}\left(\mathbf{E} \times \mathbf{H}^{*}\right) \cdot d \boldsymbol{\sigma}=\int_{V_{l}} 2 n_{l}^{\prime} n_{l}^{\prime \prime} \omega \varepsilon_{0}|\mathbf{E}|^{2} d \tau,\right.
$$

where we used Gauss's theorem for the left hand side and the right hand side stems from the material absorption in the volume containing material $l$, $V_{l}$, with $\varepsilon_{l}^{*}=\left(n_{l}^{\prime}-i n_{l}^{\prime \prime}\right)^{2}$. It now follows with Eq. (6)

$$
P_{\text {in }}-P_{\text {out }}=P_{\text {in }}\left(1-\sum_{m=1}^{Q} T_{m}\left(n_{l}^{\prime \prime}\right)\right)=-\int_{V_{l}} \omega \varepsilon_{0} n_{l}^{\prime} n_{l}^{\prime \prime}|\mathbf{E}|^{2} d \tau .
$$

Here the expression at the left hand side is the difference in power at the input and total output. Differentiation of Eq. (15) with respect to $n_{l}^{\prime \prime}$ leads to:

$$
\sum_{m=1}^{Q} 2 T_{m} \operatorname{Re}\left(\frac{\partial \ln t_{m}}{\partial n_{l}^{\prime \prime}}\right)=\sum_{m=1}^{Q}-2 T_{m} \operatorname{Im}\left(\frac{\partial \ln t_{m}}{\partial n_{l}^{\prime}}\right)=\int_{V_{l}} \omega \varepsilon_{0} n_{l}^{\prime}|\mathbf{E}|^{2} d \tau / P_{i n},
$$

where we used the analyticity of $t_{m}$ for the first equality. Taking the second equality of Eq. (16), multiplying it with $G_{l} / 2$ defined by Eq. (11) and using Eq. (13) results into

$$
\sum_{m=1}^{Q} T_{m} \tau_{g, m}^{l}=\frac{1}{2} \frac{\partial\left(n_{l} \omega\right)}{\partial \omega} \int_{V_{l}} \varepsilon_{0} n_{l}|\mathbf{E}|^{2} d \tau / P_{i n}
$$

with $V_{l}$ the volume of material $l$ and $n_{l}$ is a real quantity. The left hand side of Eq. (17) corresponds to the partial group delays summed over the output channels and weighted by the transmittances of these output channels. After summing Eq. (17) over $l$ we arrive at

$$
\begin{aligned}
& \sum_{m=1}^{Q} T_{m} \tau_{g, m}=\sum_{l=1}^{P} \frac{1}{2} \frac{\partial\left(n_{l} \omega\right)}{\partial \omega} \int_{V_{l}} \varepsilon_{0} n_{l}|\mathbf{E}|^{2} d \tau / P_{i n} \\
& =\int_{V} \varepsilon_{0}(\varepsilon+\partial(\varepsilon \omega) / \partial \omega)|\mathbf{E}|^{2} d \tau /\left(4 P_{i n}\right) .
\end{aligned}
$$

Here $V$ is the entire volume of the considered structure.

A more compact form for the above equation is obtained if the following holds

$$
\operatorname{Im} \int_{\text {surface }}\left(\mathbf{E} \times \mathbf{H}^{*}\right) \cdot d \boldsymbol{\sigma}=0,
$$

where the integral runs over the surface of the structure. If the structure contains WGs, Eq. (19) means that the corresponding modal fields have only a considerable value at the structure's surface in directions perpendicular to the propagation direction of these modes. This corresponds to WGs approaching the structure's surface perpendicularly. This is seen from the fact that for guided modes the vector $\operatorname{Im}\left(\mathbf{E} \times \mathbf{H}^{*}\right)$ is oriented along the transverse directions of a WG if it is lossless. Using Eq. (19), integrating the imaginary part of Eq. (14), now with real $\varepsilon$, over the volume of the device, and using Gauss' theorem for the left hand side of the resulting equation, it follows

$$
\int_{V} \mu_{0}|\mathbf{H}|^{2} d \tau=\int_{V} \varepsilon \varepsilon_{0}|\mathbf{E}|^{2} d \tau,
$$


and Eq. (18) can be rewritten as

$$
\sum_{m=1}^{Q} T_{m} \tau_{g, m}=\int_{V} D d \tau / \int_{A} S_{i n \perp}^{\text {Poynting }} d \sigma,
$$

where $S_{\text {in } \perp}^{\text {Poynting }}$ (with $P_{\text {in }}=\int_{A} S_{\text {in } \perp}^{\text {Poynting }} d \sigma$ ) is the component of the time-averaged Poynting vector of the incoming field perpendicular to the surface of the input area $A$ and $D$ is the total time-averaged energy density, being the sum of the time-averaged electric energy density $D_{E}$ and the time-averaged magnetic energy density $D_{H}$. The latter two are given by $[11,12]$

$$
D_{E}=\varepsilon_{0}[\partial(\varepsilon \omega) / \partial \omega]|\mathbf{E}|^{2} / 4
$$

and

$$
D_{H}=\mu_{0}|\mathbf{H}|^{2} / 4 \text {. }
$$

It is noted that in the above derivation of Eq. (21) the correct form for $D_{E}$, with material dispersion included, comes out automatically.

The left hand side of Eq. (21) corresponds to the sum of group delays weighted by their transmittances. It is seen that this sum is always positive (or zero) and that, only in the case of more than one output, at a certain output channel pulse advancement may occur, corresponding to $\tau_{g, m}<0$.

As a check to the above we note that for mode propagation in a photonic crystal WG the result given by Eq. (21) turns into

$$
v_{g}=\int_{\text {unit cell }} S_{i n \perp} d \tau / \int_{\text {unit cell }} D d \tau,
$$

with $v_{g}$ the group velocity of the mode, in agreement with the result of [13].

\section{Example to the relation on group delay}

To illustrate the above we consider an easy-reference 1D example, being a Fabry-Perot cavity consisting of a plane parallel plate with thickness $L$ and index $n_{2}$ in a surrounding medium with index $n_{1}$ (see inset of Fig. 2(a)). The device boundaries are defined by two planes just outside the cavity. The structure is excited by a plane wave coming in perpendicularly to the plate. The electrical fields are given by:

$$
\begin{aligned}
& E_{y}=E_{0}\left(e^{-i k_{0} n_{1} Z}+t_{2} e^{i k_{0} n_{1} Z}\right), \quad z<0 \\
& E_{y}=E_{0} t_{1}\left(e^{-i k_{0} n_{2}(z-L)}+r_{21} e^{i k_{0} n_{1}(z-L)}\right) /\left(1+r_{21}\right), 0<z<L \\
& E_{y}=E_{0} t_{1} e^{-i k_{0} n_{1}(z-L)}, \quad z>L
\end{aligned}
$$

The magnetic fields can be obtained from these using one of Maxwell's curl equations

$$
H_{x}=\frac{-i}{\omega \mu_{0}} \frac{\partial E_{y}}{\partial z} .
$$



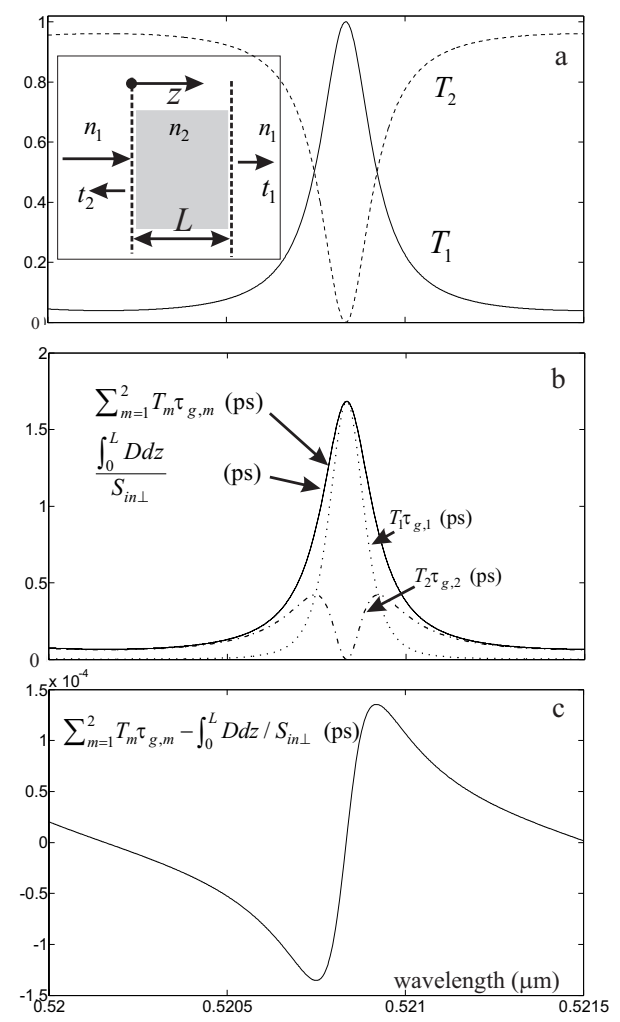

Fig. 2. Computational results for a simple Fabry-Perot cavity: a) schematic of the structure and reflectance $T_{2}$ and transmittance $T_{1}$, b) and c) dispersion curves of the indicated quantities.

The reflection coefficient for the interface between medium 2 and 1 and the modal coefficients for reflection and transmission are given by

$$
r_{21}=\frac{n_{2}-n_{1}}{n_{2}+n_{1}}, \quad t_{2}=\frac{r_{21}\left(e^{-2 i k_{0} n_{2} L}-1\right)}{1-r_{21}^{2} e^{-2 i k_{0} n_{2} L}}, t_{1}=\frac{\left(1-r_{21}^{2}\right) e^{-i k_{0} n_{2} L}}{1-r_{21}^{2} e^{-2 i k_{0} n_{2} L}},
$$

respectively.

It is noted that the reflected field coincides with the incoming field and consequently Eq. (6) does not strictly hold in general if absorption is assumed. This is seen from the expression for the relative net power per unit area at the left hand side of the cavity given by the ratio of the z-components of the time-averaged Poynting vectors of the total field and the incoming field

$$
P_{2, \text { net }} / P_{i n}=\operatorname{Re}\left[\left(1+t_{2}\right) n_{1}^{*}\left(1-t_{2}^{*}\right)\right] / n_{1}^{\prime}=1-\left|t_{2}^{2}\right|-2 t_{2}^{\prime \prime} n_{1}^{\prime \prime} / n_{1}^{\prime} .
$$

The incoming power per unit area is given by:

$$
P_{i n}=\left|E_{0}\right|^{2} k_{0} n_{1}^{\prime} /\left(2 \omega \mu_{0}\right) .
$$

It is seen from Eq. (28) that Eq. (6), and consequently the first equality of Eq. (15), and Eq. (17) and (21) derived from it, hold only if $t_{2}^{\prime \prime}=0$.

In Fig. 2(a) we have plotted the transmittances $T_{1}$ and $T_{2}$ of the cavity, assuming dispersionless materials with indices $n_{1}=1$ and $n_{2}=10$ and a cavity length of $L=10 \mu \mathrm{m}$, 
for the indicated wavelength range. The functional values of the left and right hand side of Eq. (21) are given in Fig. 2(b), showing that the corresponding curves virtually coincide. The curves have been evaluated using analytical expressions for the group delays, based on Eq. (7) using Eq. (27) and the integral over the time-averaged energy density using field expressions of Eq. (25). The difference between the two values is small (negligible for all practical purposes, see Fig. 2(c)) and, as mentioned above, equal to zero if $t_{2}^{\prime \prime}=0$, corresponding to $2 k_{0} n_{2} L=m \pi$ according to the second equality of Eq. (27), with $m$ an integer.

\section{Discussion}

In this section we will discuss how partial group delay and sensitivity can be converted into each other and that a large ratio of the energy in the material to be sensed (labeled by s) and the input power is a sufficient condition to enable a high sensitivity. We assume a structure having properties as mentioned in the beginning of section 3 and as required for Eq. (17). We consider the complex quantity $\tilde{S}_{m}^{s}$ defined by:

$$
\tilde{S}_{m}^{s} \equiv \frac{\partial \ln t_{m}}{\partial n_{s}},
$$

where $m$ denotes a channel showing a large group delay. Recalling the definitions given in sections 2 (Eq. (1)) and 3 (Eq. (13)) it is seen that the imaginary part of $\tilde{S}_{m}^{s}$ is proportional to $\tau_{g, m}^{s}$ whereas the absolute value of its real part is one half of the sensitivity according to

$$
\left|\operatorname{Re}\left(\tilde{S}_{m}^{s}\right)\right|=\left|\operatorname{Re}\left(\frac{\partial \ln t_{m}}{\partial n_{s}}\right)\right|=\frac{1}{2}\left|\frac{\partial \ln T_{m}}{\partial n_{s}}\right|=\frac{1}{2} S_{m}^{s},
$$

where the subscript and superscript of $S$ denote output channel and material, respectively. So, sensitivity and partial group delay are proportional to real and imaginary parts of the same complex quantity $\tilde{S}_{m}^{s}$. The numerical values of the two parts can be very different, as illustrated by a propagating mode in a lossless photonic crystal WG structure for which the group delay can be very high, whereas the sensitivity is zero.

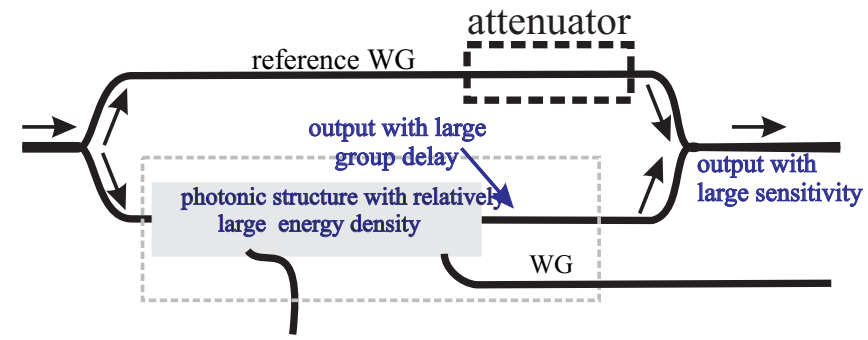

Fig. 3. Schematic of a MZI like set-up to convert partial group delay and sensitivity into each other.

Real and imaginary parts of $\tilde{S}_{m}^{s}$ can be converted into each other in, for example, a MachZehnder Interferometer (MZI)-like configuration as sketched in Fig. 3. This can be seen as follows. Assuming $3 \mathrm{~dB}$ splitters and combiners, the transmission coefficients of the excited modes at the output are

$$
t_{ \pm}=\left(t_{m} \pm t_{r e f}\right) / 2,
$$

where $t_{m}$ is the transmission coefficient of output channel $m$ of an (in principle) arbitrary photonic structure, placed in the signal branch of the MZI, $t_{r e f}$ is the transmission coefficient of the reference branch, and the symbol \pm indicates the symmetric $\left(^{+}\right)$and anti-symmetric $(-)$ modes of the output of the combined device. The latter mode may be non-guided. In the 
above the modal phase shifts, owing to transport through the connecting WGs, are assumed to be accounted for via $t_{\text {ref }}$. The attenuator in the reference branch is added to prevent unphysical blow-up (owing to division by small numbers) of the quantity defined below. Considering only the symmetric mode it now follows:

$$
\tilde{S}_{+}^{s} \equiv \frac{\partial \ln t_{+}}{\partial n_{s}}=\frac{1}{1+t_{r e f} / t_{m}^{*}} \frac{\partial \ln t_{m}}{\partial n_{s}}=\frac{1}{1+t_{r e f} / t_{m}^{*}} \tilde{S}_{m}^{s},
$$

where we assumed that the material $s$ is confined to the photonic structure. It is seen from Eq. (33) that by choosing, while scanning, the complex phase of $t_{r e f} / t_{m}^{*}$ equal to (for example) values around $\pi / 2$, and assuming that $\left|t_{m}\right|$ is not too low, that $\tilde{S}_{+}^{s}$ is approximately a complex-phase shifted version of $\tilde{S}_{m}^{s}$, converting partial group delay of the photonic structure into sensitivity of the combined MZI-like structure. The principle of the above conversion is well known and has been applied for years in MZI-based sensors (see for example [1]).

As an illustration to the above we consider a lossless micro-cavity coupled to a single mode WG as depicted in the inset of Fig. 4a. The corresponding transmission coefficient is given by [14]

$$
t_{1}=\left(\tau-e^{-i k_{0} N L}\right) / M ; M \equiv 1-\tau e^{-i k_{0} N L},
$$

where $\tau$ is the (assumingly real) transfer coefficient of the coupler formed by micro-cavity and WG. The modal index of WG and of the WG forming the micro-cavity is $N$ and $L$ is the length of one cavity roundtrip. From Eq. (34) it follows that $T_{1} \equiv\left|t_{1}\right|^{2}=1$ and, assuming for simplicity that there is no WG dispersion (i.e., $\partial N / \partial \omega=0$ ), the following equality holds for the group delay

$$
\tau_{g, 1}=i t_{1}^{*} \partial t_{1} / \partial \omega=N L\left(1-\tau^{2}\right) /\left(c|M|^{2}\right)
$$

Before discussing the conversion of group delay into sensitivity we will check the validity of Eq. (21) for this device. It is noted that the right hand side of the above expression is the product of the inverse of the group velocity of considered (dispersionless) WG $(N / C)$, the round trip length $L$ and $|A|^{2}=\left(1-\tau^{2}\right) /\left(|M|^{2}\right)$, with $A$ the ratio of the modal amplitude in the cavity and that in the input WG, according to $A=\sqrt{1-\tau^{2}} / M$. Rewriting Eq. (24) into

$$
v_{g}^{-1}=\int_{\text {cross section }} D d \tau / P_{\text {in }}=N / C
$$

and substituting the second equality above into Eq. (35) it is now seen that Eq. (21) holds for the considered device as

$$
T_{1} \tau_{g, 1}=N L\left(1-\tau^{2}\right) /\left(c|M|^{2}\right)=\int_{\text {cavity }} D d \tau / P_{\text {in }} .
$$

In Fig. (4a) we have plotted the quantity $\tilde{S}_{1}^{s}$ defined by Eq. (30), assuming practical values for the micro-cavity being $\partial N / \partial n_{s}=0.1, N=1.5$ and $L=100 \mu \mathrm{m}$, for a certain wavelength range, as derived from Eq. (34) and given by:

$$
\tilde{S}_{1}^{s}=-0.1 i k_{0} L\left(1-\tau^{2}\right) /|M|^{2} .
$$



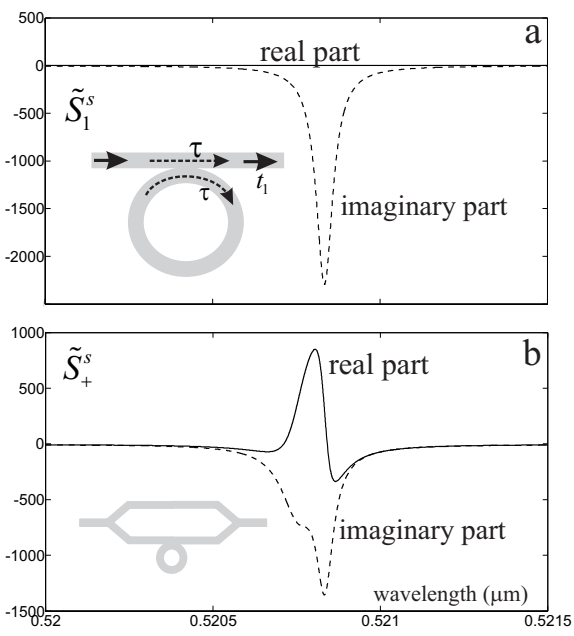

Fig. 4. Real and imaginary parts of the quantities $\tilde{S}_{1}^{s}$ (a) and $\tilde{S}_{+}^{s}$ (b) versus excitation wavelength. The insets show the considered structural lay-outs.

The quantity $\tilde{S}_{1}^{s}$ for the considered device is purely imaginary corresponding to zero sensitivity. Figure (4b) shows a plot of the quantity $\tilde{S}_{+}^{s}$ defined by Eq. (33), where we have assumed that the WGs for the MZI are identical to that of the micro-cavity (but insensitive) and we assumed $t_{\text {ref }}=\exp \left(-i k_{0} N L_{1}\right) / \sqrt{2}$, with $L_{1}=200 \mathrm{~nm}$. The choices of both attenuation (leading to $\left|t_{\text {ref }}\right|=\sqrt{2} / 2$ ) and the phase of the reference branch signal are a bit arbitrary. As we found, any value for $L_{1}$ would lead to the desired conversion (as may be seen with Eq. (33)) as around resonances the phase of $t_{1}$ varies as a function of the wavelength so that for any value of $L_{1}$ the real part of $\tilde{S}_{+}^{s}$ will be comparable in magnitude to the imaginary part of $\tilde{S}_{1}^{s}$. From Fig. 4 it is indeed seen that the conversion is considerably and that the real part of $\tilde{S}_{+}^{s}$ is of the order of magnitude of the imaginary part of $\tilde{S}_{1}^{s}$.

Considering the above conversion from partial group delay to sensitivity and also using Eq. (17) the following can be concluded: If in the material to be sensed (s) of a certain photonic structure the ratio of time-averaged energy and input power is high (corresponding to a large partial group delay), an output channel $(m)$ may be selected for which $T_{m} \tau_{g, m}^{s}$ is large which may be converted into a large sensitivity. It is noted that the presented theory does not allow us to state that a large energy in the sensed material relative to the input power is always a necessary condition for a high sensitivity although one might expect such by intuition.

\section{Conclusions}

The relevance of a definition for sensitivity in refractometric sensing on the basis of the relative change of the transmittance divided by the change of the probed refractive index has been discussed. It is argued that the above definition, with a sensitivity which is directly related to the limit of detection, is more adequate than one based on spectral shift per refractive index unit. The latter definition may lead to a large overestimation of the sensing performance as is shown via two examples. 
A relation has been derived for non-absorbing structures showing the equality of the weighted (by the transmittance) group delays, summed over the output channels, and the ratio of the total optical energy and the input power. A nearly equivalent equality holds if partial group delays and partial energy are considered, where partial refers to a sample material of the structure. Next it is shown that partial group delay and sensitivity of a certain photonic structure can be approximately converted into each other. Combing the above findings it follows that a large energy density in the probed material relative to the input power enables the construction of a highly sensitive device.

\section{Acknowledgments}

The authors thank P.V. Lambeck and T.P. Valkering for stimulating and helpful discussions on the subject of this paper.

\section{References and links}

1. P. V. Lambeck, "Integrated optical sensors for the chemical domain,” Meas. Sci. Technol. 17, R93-R116 (2006).

2. C. McDonagh, C. S. Burke, and B. D. MacCraith, “Optical Chemical Sensors,” Chem. Rev. 108, 400-422 (2008).

3. I. M. White and X. Fan, "On the performance quantification of resonant refractive index sensing," Opt. Express 16, 1022-1028 (2008).

4. S. V. Pham, M. Dijkstra, A. J. F. Hollink, L. J. Kauppinen, R. M. de Ridder, M. Pollnau, P. V. Lambeck, and H. J. W. M. Hoekstra, “On-chip bulk-index concentration and direct, label-free protein sensing utilizing an optical grated-waveguide cavity,” Sens. Actuator B - Chem. 174, 602-608 (2012).

5. P. Pfeifer, U. Aldinger, G. Schwotzer, S. Diekmann, and P. Steinrücke, "Real time sensing of specific molecular binding using surface plasmon resonance spectroscopy,” Sens. Actuator B 54, 166-175 (1999)

6. M. Vala, K. Chadt, M. Piliarik, and J. Homola, "High-performance compact SPR sensor for multi-analyte sensing,” Sens. Actuator B 148, 544-549 (2010).

7. T. Claes, W. Bogaerts, and P. Bienstman, "Experimental characterization of a silicon photonic biosensor consisting of two cascaded ring resonators based on the Vernier-effect and introduction of a curve fitting method for an improvement of the detection limit,” Opt. Express 18, 22747-22761 (2010).

8. T. Claes, W. Bogaerts, and P. Bienstman, "Vernier-cascade label-free biosensor with integrated arrayed grating for wavelength interrogation with low-cost broadband source,” Opt. Lett. 36, 3320-3322 (2011).

9. K. Naganuma, K. Mogi, and H. Yamada, "Group-delay measurement using the Fourier transform of an interferometric cross correlation generated by white light,” Opt. Lett. 15 393-395 (1990).

10. W. C. Chew, Waves and fields in inhomogeneous media (IEEE Press Series on Electromagnetic Waves, Series Editor D. G. Dudley, IEEE Press, New York, 1995).

11. L. Brillouin, Wave propagation and group velocity (Academic Press, New York and London, 1960).

12. J. B. Khurgin, "Slow light in various media: a tutorial,” Adv. Opt. and Phot. 2, 287-318 (2010).

13. P. Yeh, "Electromagnetic propagation in birefringent layered media,” J. Opt. Soc. Am. 69, 742-756 (1979).

14. H. J. W. M. Hoekstra, P. V. Lambeck, H. P. Uranus, T. M. Koster, "Relation between noise and resolution in integrated optical refractometric sensing,” Sens. Actuator B-Chem. 134, 702-710 (2008) 